\title{
Spatial time-frequency distribution of cross term-based direction-of-arrival estimation for weak non-stationary signal
}

\author{
Shuai Shao ${ }^{1,2}$, Aijun Liu', Changjun Yu', Hongjuan Yang ${ }^{1,2^{*}}$ (D), Yong Li ${ }^{2,3}$ and Bo Li ${ }^{1,2}$
}

\begin{abstract}
In the radar array signal processing direction of arrival (DOA), the estimation of weak non-stationary signal is an important and difficult problem when both strong and weak signals are coexisting particularly because the weak non-stationary signals are often submerged in noise. In this paper, we proposed a novelty method to estimate the direction of arrival (DOA) of weak non-stationary signal in scenario for strong non-stationary interference signals and Gaussian white noise. The method utilizes spatial time-frequency distribution (STFD) of cross terms rather than suppressing cross terms in time-frequency analysis. The STFD of cross terms are introduced as an alternative matrix, which is similar to data covariance matrix in multiple signal classification (MUSIC), for the DOA estimation of a weak non-stationary signal. The cross-term amplitude of the strong and weak signals is usually above the noise and is easier to use than the auto-term of the weak signal. In the cross term, the information of the weak signal is included, and the auto-term of these weak signals is difficult to extract directly. The ability to incorporate the STFD of cross terms empowers information about a weak non-stationary signal for DOA estimation, leading to improved signal estimates for direction finding. The method based on the STFD of cross terms for DOA estimation of the weak non-stationary signal is revealed to outperform the time-frequency MUSIC and traditional MUSIC algorithm by simulation, respectively. This method has the advantages of the time-frequency direction finding method and also deals with the situation of weak signals. When the strong and weak signals exist at the same time and the two angles are similar, the cross-terms can be used to perform DOA estimation on the weak signal.
\end{abstract}

Keywords: DOA estimation, Time-frequency analysis, Spatial time-frequency distribution (SFTD), Cross terms

\section{Introduction}

Among numerous non-stationary signals that arise in many radars [1] and communication [2, 3], instantaneous frequency (IF) signals, for instance, linear frequency modulated (LFM) signals, have obvious time-frequency characteristics which are continuous and decided the location. Similar to the time-frequency signatures, the spatial signature of the signal source also includes significant information about the signal source [4]. It ensures signal source identification due to the respective angle position received from a receiver antenna array, which is the directions-ofarrival. The characterization of DOA is viewed as steering

\footnotetext{
* Correspondence: hjyang@hit.edu.cn

'School of Information Science and Engineering, Harbin Institute of

Technology (Weihai), Weihai, China

${ }^{2}$ Science and Technology on Communication Networks Laboratory,

Shijiazhuang, China

Full list of author information is available at the end of the article
}

vectors in which the source signal demonstrates a difference of the signal phase over every sensor antennas when electromagnetic wave covers the receiver antenna array [5]. Time-frequency (T-F) analysis plays important roles in the DOA estimation of the non-stationary signal [6].

Time-frequency analysis enables to process nonstationary signals overlying in both frequency and time domains in which windowing- and filtrating-based means cannot separate the different signal source components $[7,8]$. For analyzing the non-stationary signals, such as LFM signals, time-frequency signal representations and analyses are necessary [9]. We engage in the class of signals where the instantaneous frequency particularly or basically determines the time-frequency signatures of the signal source. A successful application of time-frequency distribution desires prior knowledge for the signal source in order that the most advisable 
distribution is preferred [10]. In this article, WignerVille distribution (WVD) is considered as the timefrequency distribution representation since it provides the most energy concentration in time-frequency domains, displays the non-stationary properties of the signal, and satisfies the marginal conditions [11]. Combining the time-frequency and spatial characteristic is accomplished into a framework named STFDs in time-frequency MUSIC (TF-MUSIC) [12]. This structure applies the signal location characteristic and energy concentration for increasing signal-to-noise ratio (SNR) and source signal identification before achieving the highresolution direction-of-arrival estimation [13]. The framework desires the calculations of the WVD from the data obtained at each sensor, for instance, auto-terms of WVD and the cross terms of WVD between sensor antennas.

When the analyzed signal includes more than one signal source component, WVD performs signal autoterms as positive magnitudes at their instantaneous frequency areas and the cross terms as oscillatory magnitudes along the geometrical middle point of autoterms [14]. The cross-term problem of the WVD was first indicated in [15]. The suppression of cross terms is the core problem of bilinear time-frequency transformation. The references are abundant studies to suppress the cross terms and increase the time-frequency resolution. A method combined of the Hough transform (HT) and the Wigner-Ville distribution (WVD) performs cross term suppression $[14,16]$. Another study researched the blind source separation approach based on the use of time-frequency analysis to eliminate cross terms in WVD [17]. The main purpose of research in [18] is to accomplish the high-resolution and the maximal cross-term contraction with the desirable diagonal or off-diagonal peculiarity of time-frequency distribution matrices in blind source separation applications. Researchers proposed a method named standardization of the pseud-quadratic form to suppress the cross terms in [19]. In order to suppress the cross term, Zuo et al. further propose a smoothed high-resolution time-frequency rate representation (SHR-TFRR) via utilizing an FR window to the highresolution-time frequency rate representation, which is expressed in the convolution form [20,21]. A pure idea is offered by Aiordachioaie and Popescu, as starting initial point to compose an approach to suppress the cross terms and to attain an exact image of WVD, including only the auto-terms [22]. A new method is offered by $\mathrm{Wu}$ and $\mathrm{Li}$ to suppress cross terms in the WVD of linear frequency modulation signals with multicomponent [23].

The existence of cross terms is difficult to be avoided. There are some researches in the references that take full use of cross terms in WVD in different research fields. Bird song syllable classification is realized using cross terms of Wigner-Ville ambiguity function in [24]. The Wigner distribution achieves artifacts well known as cross terms that are contemplated and undesirable in some situations; nevertheless, it can be utilized to ascertain the existence of greatly little signal source terms, in this case, VLPs terms [25]. Using the ambiguity domain interpretation, Jeong and Williams explored the theory of the cross terms (or interferences) in spectrograms [26]. A blind source separation technology applying both cross terms and auto-terms in the timefrequency distributions of the source signals was considered by Belouchrani et al. in [27]. As an application, Fadaili et al. showed that the source separation can be accomplished via exploiting one of these algorithms to a set of spatial quadratic time-frequency distribution matrices corresponding merely to the named cross terms and/or to the named auto-terms [28]. When the cross term is considered from different perspectives, the cross term accurately reflects the relationship between multiple signal components [29]. When the energy difference between non-stationary signals is large, specifically in the situation of low signal-to-noise ratio, a weak non-stationary signal may be buried in the noise. At this point, the weak non-stationary signal is the desired signal. It is difficult or almost impossible to extract the auto-term of the weak non-stationary signal. The cross-term amplitude of strong and weak nonstationary signal did not decrease significantly. In general, the cross terms contain sufficient information of weak non-stationary signal for its DOA estimation. Therefore, in the case that both strong and weak nonstationary signals existing simultaneously, the cross terms of STFDs are used to obtain DOA of the desired weak non-stationary signal in this paper.

This paper includes some sections. Section 2 reviews spatial time-frequency distribution in TF-MUSIC. In Section 3, cross-term selection procedures of STFDs are introduced. The analytical results are used in this section in order to examine the proposed method performance. Several simulations are offered in Section 4. Section 5 gives conclusions.

\section{Related works}

In narrowband signal array processing, because $n$ source signals access on a $m$-element uniform linear array, the received data formula

$$
\mathbf{x}(t)=\mathbf{y}(t)+\mathbf{n}(t)=\mathbf{A}(\theta) \mathbf{d}(t)+\mathbf{n}(t)
$$

is frequently presumed, where the $m \times n$ spatial matrix $\mathbf{A}(\theta)=\left[\mathbf{a}\left(\theta_{1}\right), \mathbf{a}\left(\theta_{2}\right), \ldots, \mathbf{a}\left(\theta_{n}\right)\right]$ implies the steering matrix. In the direction-of-arrival estimate situations, we expect A to be a well-known characteristic, and each column of matrix $\mathbf{A}$ associated with a single signal direction drives 
a distinct orientation. The analytical operation in this article does not rely upon any special matrix A structure characteristic. Because of the synthesis of the source signals at every antenna, the parts of the $m \times 1$ data vector $\mathbf{x}(t)$ are multi-component source signals; nevertheless, every source signal $d_{i}(t)$ of the source signal vector $\mathbf{d}(t)$ is often a single signal component. $\mathbf{n}(t)$ is an additive zero-mean white complex noise vector in which elements are presumed as temporal and spatial stationary random processes which are independent from the source signals.

In (1), it is presumed that the quantity $m$ of sensors is more than the quantity $n$ of source signals. Furthermore, matrix A has a column full rank because it involves that the steering vectors associated with $n$ different directions of arrival have linear independence relationship. We furthermore presumed that the data covariance matrix

$$
\mathbf{R}_{\mathbf{x} \mathbf{x}}=\mathrm{E}\left[\mathbf{x}(t) \mathbf{x}^{\mathrm{H}}(t)\right]
$$

is not singular and that the processing duration includes $N$ snapshots $(N>m)$, where superscript $\mathrm{H}$ implies conjugate transpose, and $\mathrm{E}(\cdot)$ implies the statistical expectation operator. From the above presumptions, the data correlation matrix is provided by

$$
\mathbf{R}_{\mathbf{x} \mathbf{x}}=\mathrm{E}\left[\mathbf{x}(t) \mathbf{x}^{\mathrm{H}}(t)\right]=\mathbf{A} \mathbf{R}_{\mathbf{d d}} \mathbf{A}^{\mathrm{H}}+\sigma^{2} \mathbf{I}
$$

where $\mathbf{R}_{\mathbf{d d}}=\mathrm{E}\left[\mathbf{d}(t) \mathbf{d}^{\mathrm{H}}(t)\right]$ is the signal correlation matrix, $\sigma^{2}$ is the noise power at every sensor antenna, and $\mathbf{I}$ implies the identity matrix. Let $\lambda_{1}>\lambda_{2}>\ldots>\lambda_{n}>\lambda_{n+}$ $1=\lambda_{n+2}=\ldots=\lambda_{m}=\sigma^{2}$ imply the characteristic values of $\mathbf{R}_{\mathbf{x x}}$. The $\lambda_{i}, i=1, \ldots, n$ are individual. The eigenvectors corresponding to $\lambda_{1}, \ldots, \lambda_{n}$ make up the columns of the matrix $\mathbf{S}=\left[\mathbf{s}_{1}, \ldots, \mathbf{s}_{n}\right]$, and those associated with $\lambda_{n+1}, \ldots$, $\lambda_{m}$ constitute the matrix $\mathbf{G}=\left[\mathbf{g}_{1}, \ldots, \mathbf{g}_{m-n}\right]$. Because the column vectors of $\mathbf{A}$ and $\mathbf{S}$ constitute their subspace, $\mathbf{A}^{\mathrm{H}} \mathbf{G}=\mathbf{0}$.

In effect, $\mathbf{R}_{\mathbf{x x}}$ is not known and could be approximated via the applicable data snapshots $\mathbf{x}(i), i=1, \ldots, N$. The approximated data covariance matrix is provided from

$$
\hat{\mathbf{R}}_{\mathbf{x x}}=\frac{1}{N} \sum_{i=1}^{N} \mathbf{x}(i) \mathbf{x}^{\mathrm{H}}(i)
$$

Let $\left\{\hat{\mathbf{s}}_{1}, \ldots, \hat{\mathbf{s}}_{n}, \hat{\mathbf{g}}_{1}, \ldots, \hat{\mathbf{g}}_{m-n}\right\}$ imply the unit-norm eigenvectors of $\hat{\mathbf{R}}_{\mathbf{x x}}$ that are arranged according to a descending order of the corresponding eigenvalues and make $\hat{\mathbf{S}}$ and $\hat{\mathbf{G}}$ imply the matrices determined by the set of vectors $\left\{\hat{\mathbf{s}}_{i}\right\}$ and $\left\{\hat{\mathbf{g}}_{i}\right\}$, severally. We retrospect that the DOAs can be evaluated by the traditional MUSIC approach via resolving the $n$ values of $\theta$ for which the subsequent spatial spectrum is performed by maximization operation [30]:

$$
f_{\mathrm{MU}}(\theta)=\frac{1}{\mathbf{a}^{\mathrm{H}}(\theta) \hat{\mathbf{G}} \hat{\mathbf{G}}^{\mathrm{H}} \mathbf{a}(\theta)}
$$

where $\mathbf{a}(\theta)$ is the steering vector associated with $\theta$.

We then survey the concept and fundamental peculiarities of the STFDs. In this article, we straightforwardly examine a kind of Cohen's class, specially, the WignerVille distribution (WVD) as well as its characteristic. The discrete formula of WVD of a signal source $x(t)$, via an odd length $L$ rectangular window, is given by

$$
D_{x x}\left(k_{t}, k_{f}\right)=\sum_{k_{\tau}=-(L-1) / 2}^{(L-1) / 2} x\left(k_{t}+k_{\tau}\right) x^{*}\left(k_{t}-k_{\tau}\right) e^{-j 4 \pi k_{f} k_{\tau}}
$$

where * implies complex conjugate. The integral of the product of the conjugate of the signal reflects the energy distribution of the signal in the time and frequency dimensions. Formula (6) reflects the details of the signal energy distribution. The spatial WVD matrix is attained via changing $x(t)$ by the data snapshot vector $\mathbf{x}(t)$

$$
\mathbf{D}_{\mathbf{x x}}\left(k_{t}, k_{f}\right)=\sum_{k_{\tau}=-(L-1) / 2}^{(L-1) / 2} \mathbf{x}\left(k_{t}+k_{\tau}\right) \mathbf{x}^{\mathrm{H}}\left(k_{t}-k_{\tau}\right) e^{-j 4 \pi k_{f} k_{\tau}} .
$$

Replacing (1) into (7), we attain

$$
\begin{aligned}
\mathbf{D}_{\mathbf{x x}}\left(k_{t}, k_{f}\right)= & \mathbf{D}_{\mathbf{y y}}\left(k_{t}, k_{f}\right)+\mathbf{D}_{\mathbf{y n}}\left(k_{t}, k_{f}\right) \\
& +\mathbf{D}_{\mathbf{n y}}\left(k_{t}, k_{f}\right)+\mathbf{D}_{\mathbf{n n}}\left(k_{t}, k_{f}\right) .
\end{aligned}
$$

We note that $\mathbf{D}_{\mathbf{y y}}\left(k_{t}, k_{f}\right), \mathbf{D}_{\mathbf{y n}}\left(k_{t}, k_{f}\right), \mathbf{D}_{\mathbf{n y}}\left(k_{t}, k_{f}\right)$, and $\mathbf{D}_{\mathbf{n n}}\left(k_{t}, k_{f}\right)$ are matrices of $m \times m$ dimension. Due to the uncorrelated noise and signal presumption and the zeromean noise property, the mathematic expectation of the cross-term STFD matrices between the noise and signal vectors equal to zero, such as $\mathrm{E}\left[\mathbf{D}_{\mathbf{y n}}\left(k_{t}, k_{f}\right)\right]=\mathbf{0}$ and $\mathrm{E}\left[\mathbf{D}_{\mathbf{n y}}\left(k_{t}, k_{f}\right)\right]=\mathbf{0}$, and it pursues that

$$
\begin{gathered}
\mathrm{E}\left[\mathbf{D}_{\mathbf{x x}}\left(k_{t}, k_{f}\right)\right]=\mathrm{E}\left[\mathbf{D}_{\mathbf{y y}}\left(k_{t}, k_{f}\right)\right]+\mathrm{E}\left[\mathbf{D}_{\mathbf{n n}}\left(k_{t}, k_{f}\right)\right] \\
=\mathbf{A E}\left[\mathbf{D}_{\mathbf{d d}}\left(k_{t}, k_{f}\right)\right] \mathbf{A}^{\mathrm{H}}+\sigma^{2} \mathbf{I}
\end{gathered}
$$

where the signal source time-frequency distribution matrix

$$
\mathbf{D}_{\mathbf{d d}}\left(k_{t}, k_{f}\right)=\sum_{k_{\tau}=-(L-1) / 2}^{(L-1) / 2} \mathbf{d}\left(k_{t}+k_{\tau}\right) \mathbf{d}^{\mathrm{H}}\left(k_{t}-k_{\tau}\right) e^{-j 4 \pi k_{f} k_{\tau}}
$$

is of $n \times n$ dimension matrix. For signal array processing researches, the hybrid matrix $\mathbf{A}$ includes the azimuth and projects the auto-terms and cross terms in timefrequency distributions of the source signals to the auto- 
terms and cross terms in time-frequency distributions of the received data.

Formula (9) is comparable to the other formula that has been generally utilized for direction finding problems, involving the source signal covariance matrix to the data spatial covariance matrix. From the above production, the covariance matrices are reestablished via the STFD matrices. The reestablished formulas for traditional array signal processing could be used, and core problems for various situations of array processing, especially those addressing non-stationary signal situations, can be addressed by bilinear transformations. It is prominent that (9) suits for every point. For reducing the noise influence and ensure the column full rank character for the involved matrix, many time-frequency points are used, contrary to a single one. Joint diagonalization [31] and the time-frequency averaging approach become the two key technologies that have been utilized for the objective [7, 13]. In this article, we merely use averaging operation for many time-frequency points.

The time-frequency distribution transforms the onedimensional time domain source signals into the twodimensional time-frequency domain source signals. The time-frequency distribution characteristic of accumulating the incoming signal while widening the noise to the integrated time-frequency domain enhances the efficient SNR. Next, we calculate the signal subspace and noise subspace projection through a finite snapshot quantity of data. In the situation where the STFD matrices are averaged for the time-frequency signatures, we consider those $N-L+1$ time-frequency distribution points. The result is provided via

$$
\hat{\mathbf{D}}=\frac{1}{(N-L+1)} \sum_{i=1}^{N-L+1} \mathbf{D}_{\mathbf{x x}}\left(i_{t}, i_{f}\right)
$$

The unit-norm eigenvectors corresponding to $\hat{\lambda}_{1}^{\mathrm{tf}}, \ldots$, $\hat{\lambda}_{n_{0}}^{\mathrm{tf}}$ are implied by the columns of $\hat{\mathbf{S}}^{\mathrm{tf}}=\left[\hat{\mathbf{s}}_{1}^{\mathrm{tf}}, \ldots, \hat{\mathbf{s}}_{n_{0}}^{\mathrm{tf}}\right]$, and those associated with $\hat{\lambda}_{n_{0}+1}^{\mathrm{tf}}, \ldots, \hat{\lambda}_{m}^{\mathrm{tf}}$ are implied by the columns of $\hat{\mathbf{G}}^{\mathrm{tf}}=\left[\hat{\mathbf{g}}_{1}^{\mathrm{ff}}, \ldots, \hat{\mathbf{g}}_{m-n_{0}}^{\mathrm{tf}}\right]$. The superscript $\mathrm{tf}$ implies that the associated term is inferred from the matrix $\hat{\mathbf{D}}$. Parallelly, for time-frequency MUSIC with $n_{0}$ source signals considered, the DOAs are confirmed via confirming the $n_{0}$ peaks of the spatial spectrum which are determined from the signals' time-frequency domain:

$$
f_{\mathrm{MU}}^{\mathrm{tf}}(\theta)=\frac{1}{\mathbf{a}^{\mathrm{H}}(\theta) \hat{\mathbf{G}}^{\mathrm{tf}} \hat{\mathbf{G}}^{\mathrm{tfH}} \mathbf{a}(\theta)} .
$$

\section{Method}

The virtues of time-frequency-based DOA estimation approach may merely be realized when suitable time- frequency points are considered in the STFD matrices. The key point of this kind of method is how to choose the suitable time-frequency points. The purpose of this paper is to estimate the DOA of weak non-stationary source signals based on cross terms of spatial time-frequency distribution in the presence of strong non-stationary signal interference. In the STFD framework, the source signal time-frequency characteristics should not be highly overlapping. The source STFD matrix is

$$
\mathbf{D}_{\mathbf{d d}}\left(k_{t}, k_{f}\right)=\left(\begin{array}{cc}
D_{d_{1} d_{1}}\left(k_{t}, k_{f}\right) & D_{d_{1} d_{2}}\left(k_{t}, k_{f}\right) \\
D_{d_{2} d_{1}}\left(k_{t}, k_{f}\right) & D_{d_{2} d_{2}}\left(k_{t}, k_{f}\right)
\end{array}\right)
$$

The two source signals, $d_{1}\left(k_{t}\right)$ and $d_{2}\left(k_{t}\right)$, are LFM signals. The following four types of time-frequency points are discussed. The first-type time-frequency points are associated with signal auto-terms merely. For those points, the source signal time-frequency distribution matrix has a rank-one diagonal mathematics structure. The second-type time-frequency points are associated with source signal cross-terms merely. For the points, the signal time-frequency distribution matrix is off-diagonal matrix. That is that the matrix is considered to be off-diagonal because their diagonal elements equal to zeros. The third-type timefrequency points are associated with both source signal auto-terms and cross terms. For the points, the signal time-frequency distribution matrix has no obvious algebraic specific structure which can be immediately used. The source signal cross terms and source signal auto-terms are inexistence in the fourth timefrequency points.

The diagonal and off-diagonal mathematics structures of the first- and second-type T-F points are frequently destroyed when the source signals are mixed. The first-, second-, and third-type T-F points are significant to the DOA estimation problem. The fourth should be abandoned because they do not have any effect in this situation. In this paper, we exploit cross terms of spatial time-frequency distribution to perform DOA estimation of weak non-stationary signals when there are both strong and weak non-stationary signals.

Because of the fact that in the first-type timefrequency points, $\mathbf{D}_{\mathbf{d d}}\left(k_{t}, k_{f}\right)$ have a high outstanding algebraic structure which is a rank-one diagonal matrix, an outstanding mean to solve the directionof-arrival estimation problem will become to utilize matrix decomposition technology, which is a traditional technology for DOA estimation. Yet, the procedure of the automatic time-frequency point's selection, in the general situation, is difficult. 
Complicated time-frequency point selection technologies will be usually needed, as studied in the following.

Under the above condition, the STFDs have the structure as follows:

$$
\mathbf{D}_{\mathbf{x x}}\left(k_{t}, k_{f}\right)=\mathbf{A D}_{\mathbf{d d}}\left(k_{t}, k_{f}\right) \mathbf{A}^{\mathrm{H}}+\mathbf{D}_{\mathbf{n n}}\left(k_{t}, k_{f}\right),
$$

Consider an $n \times m$ matrix $\mathbf{W}$, named a whitening matrix, in order that WA implies a unitary matrix and implies U. It is

$$
(\mathbf{W A})(\mathbf{W A})^{\mathrm{H}}=\mathbf{U U}^{\mathrm{H}}=\mathbf{I},
$$

Pre- and post-multiplying $\mathbf{D}_{\mathbf{x x}}\left(k_{t}, k_{f}\right)$ by $\mathbf{W}$ results in the whitened matrix, defined as

$$
\begin{aligned}
\underline{\mathbf{D}}_{\mathbf{x x}}\left(k_{t}, k_{f}\right)= & \mathbf{W D}_{\mathbf{x x}}\left(k_{t}, k_{f}\right) \mathbf{W}^{\mathrm{H}} \\
= & \mathbf{U D}_{\mathbf{d d}}\left(k_{t}, k_{f}\right) \mathbf{U}^{\mathrm{H}} \\
& +\mathbf{W D}_{\mathbf{n n}}\left(k_{t}, k_{f}\right) \mathbf{W}^{\mathrm{H}},
\end{aligned}
$$

where the second equation results from the $\mathbf{W}$ concept and (14). Distinctly, the whitening process results in a linear model in which a unitary mixed matrix is structured. In a whitened situation, some technologies use trace invariance in the matrix for unitary transform, taking it likely to judge the existence of signal cross terms. One technology [27] introduces that for the second-type $\mathrm{T}-\mathrm{F}$ points, consider matrices that verify

$$
\frac{\operatorname{trace}\left\{\underline{\mathbf{D}}_{\mathbf{x x}}\left(k_{t}, k_{f}\right)\right\}}{\left\|\underline{\mathbf{D}}_{\mathbf{x x}}\left(k_{t}, k_{f}\right)\right\|}<\varepsilon
$$

where trace $\{\cdot\}$ implies matrix mathematics trace, $\|\cdot\|$ implies mathematics Frobenius norm, and $\varepsilon$ is a userdetermined positive small value. For a noise condition, the selection procedure of time-frequency points of peak power (the first- and second-type time-frequency points) may develop a severe problem when the source signals are nearly submerged in noise. This is achieved by averaging the STFDs of cross terms to solve this problem.

\section{Results and discussion}

Assume an eight-sensor uniform linear array with a half wavelength separation between each element and an observation duration of 512 samples. The two linear frequency modulation signal components are transmitted from two source signals located at angles $\theta_{1}$ and $\theta_{2}$. The initiated and finished normalized frequencies of the source signal from $\theta_{1}=30^{\circ}$ are $f_{\mathrm{s} 1}=0$ and $f_{\mathrm{e} 1}=0.3$, whereas the homologous second frequencies for another source from $\theta_{2}=40^{\circ}$ are $f_{\mathrm{s} 2}=0.2$ and $f_{\mathrm{e} 2}=0.5$, severally. WVD is utilized to calculate the time-frequency distribution, and time-frequency averaging is utilized to build the noise subspace. The input SNR of $\theta_{1}$ is $5 \mathrm{~dB}$, whereas the incoming SNR of $\theta_{2}$ is $-5 \mathrm{~dB}$. Figure 1 shows the time-frequency spectrum of two LFM signals. From Fig. 1, the auto-term of the weak signal cannot be

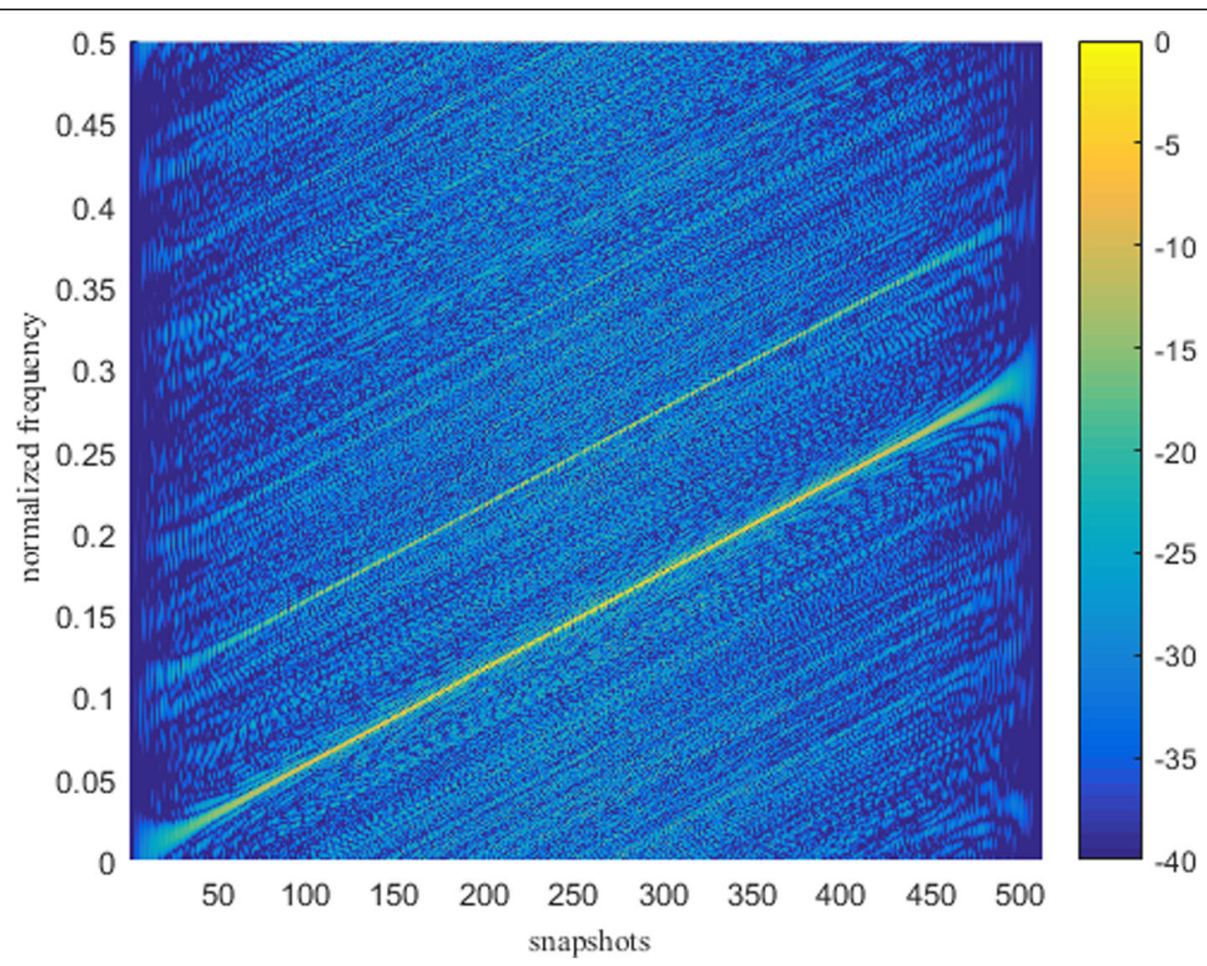

Fig. 1 T-F spectrum of two LFM signals 


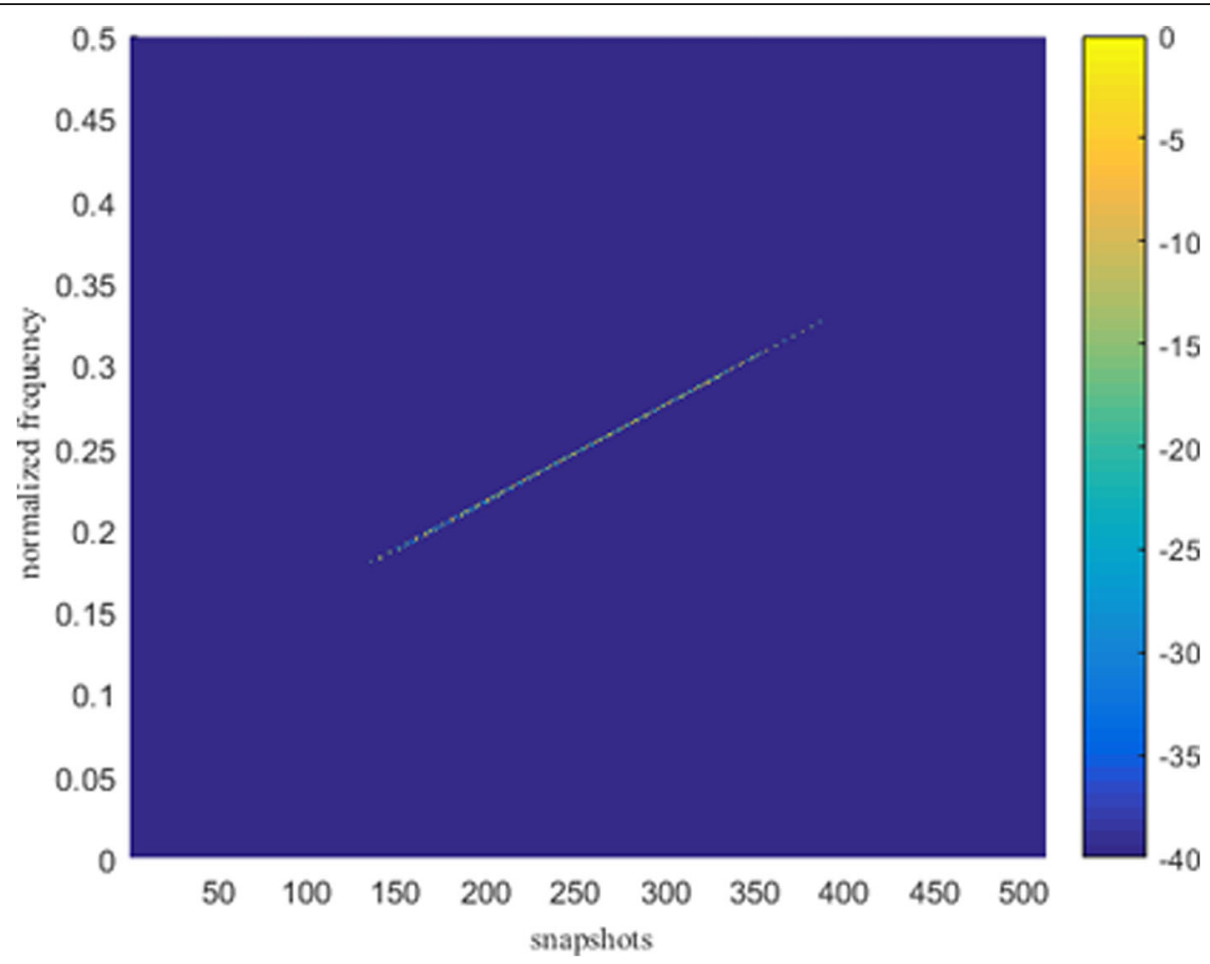

Fig. 2 Cross terms of T-F spectrum

obtained on the time-frequency spectrum because of the submergence in noise. However, the cross terms in time-frequency plane can observe the normalized frequencies of which is approximately from 0.1 to 0.4. As shown in Fig. 2, the cross terms are extracted by the method mentioned above for the DOA estimation of the weak non-stationary signal. The DOA of strong and weak signals is estimated by cross terms in Fig. 3, which are approximately $30^{\circ}$ and $40^{\circ}$, respectively.

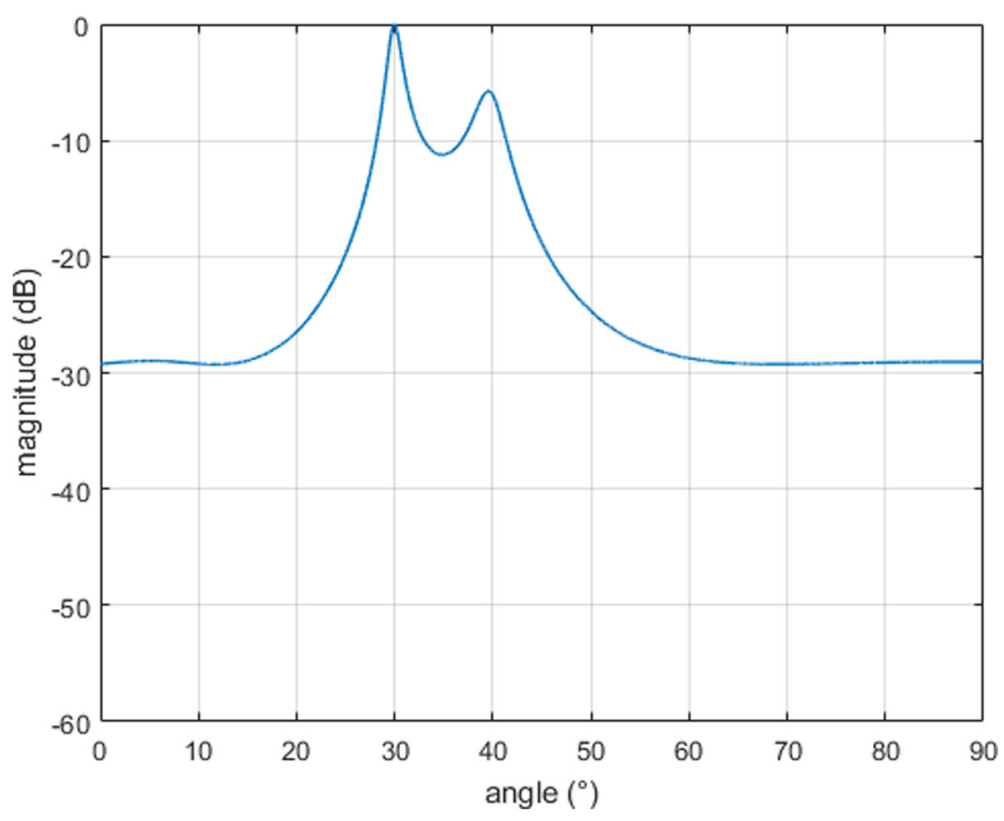

Fig. 3 The estimated spatial spectrum 

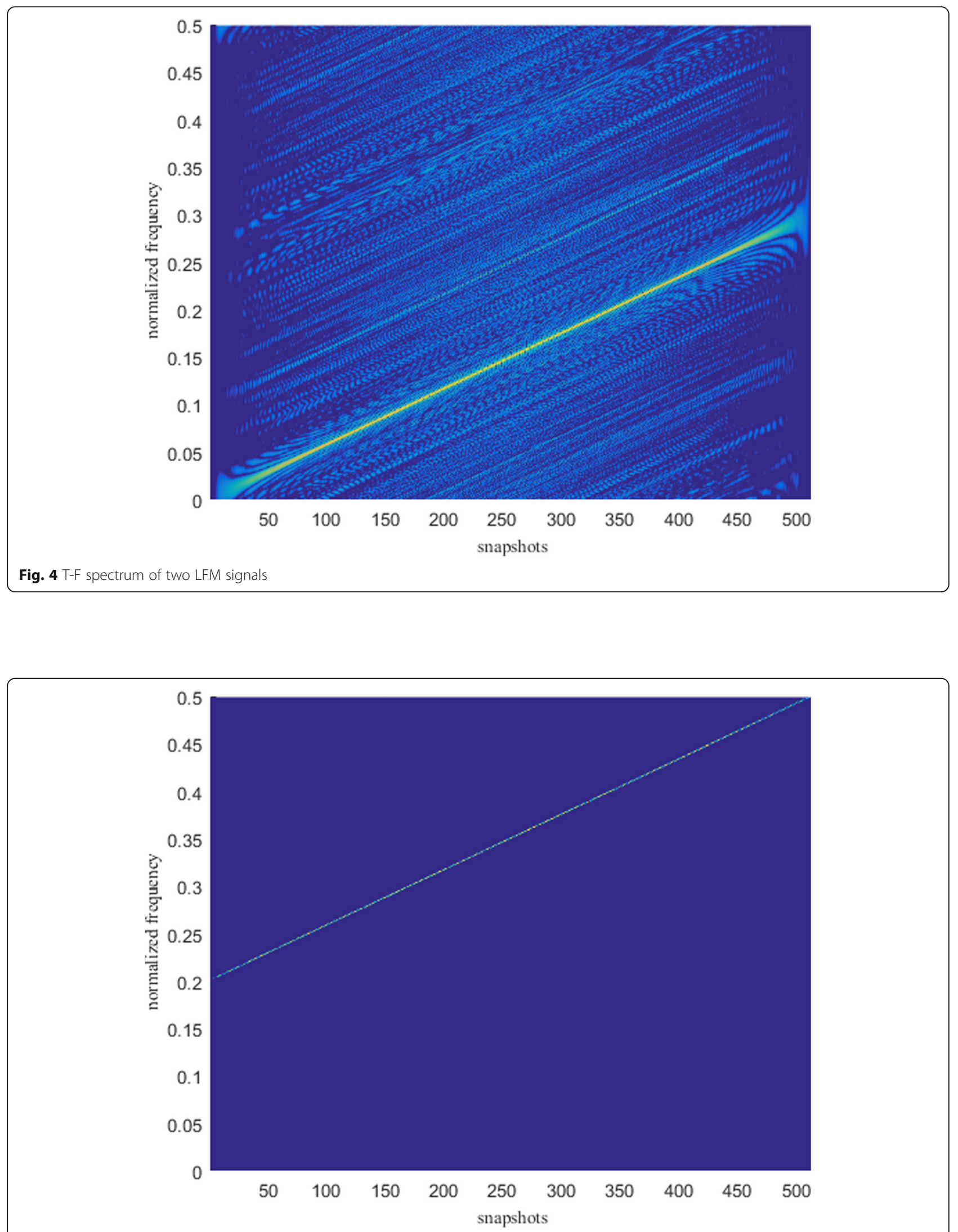

Fig. 5 Auto-terms of weak signal 


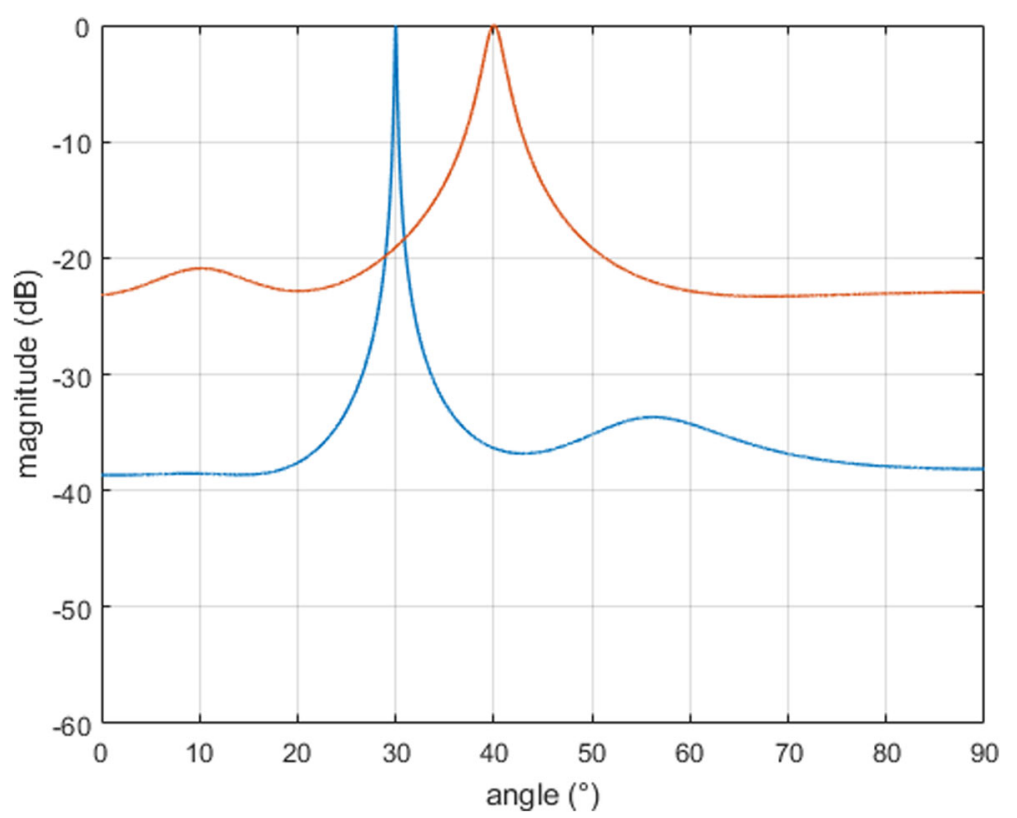

Fig. 6 The estimated spatial spectrum

If the SNR difference of the strong and weak signals increases further, the DOA of the weak signal cannot be directly obtained from the cross terms. The input SNR of $\theta_{1}$ is $10 \mathrm{~dB}$, whereas the input SNR of $\theta_{2}$ is $-10 \mathrm{~dB}$. The cross term is utilized from another perspective. Firstly, the position of the auto-terms of the strong signal and cross terms in the time-frequency domain is estimated. Then the position of the auto-terms of the weak signal is fitted according to the two positions in order to improve the SNR of the weak non-stationary signals. The spatial time-frequency distribution matrixes are extracted from the position of the weak signal for the DOA estimation. Figure 4 shows the T-F-spectrum of two linear frequency modulation signal components. Although the cross terms are no longer obvious on the time-frequency plane, they can also be extracted according to the characteristics of the matrix. In Fig. 5, the auto-terms of the weak non-stationary signal are fitted

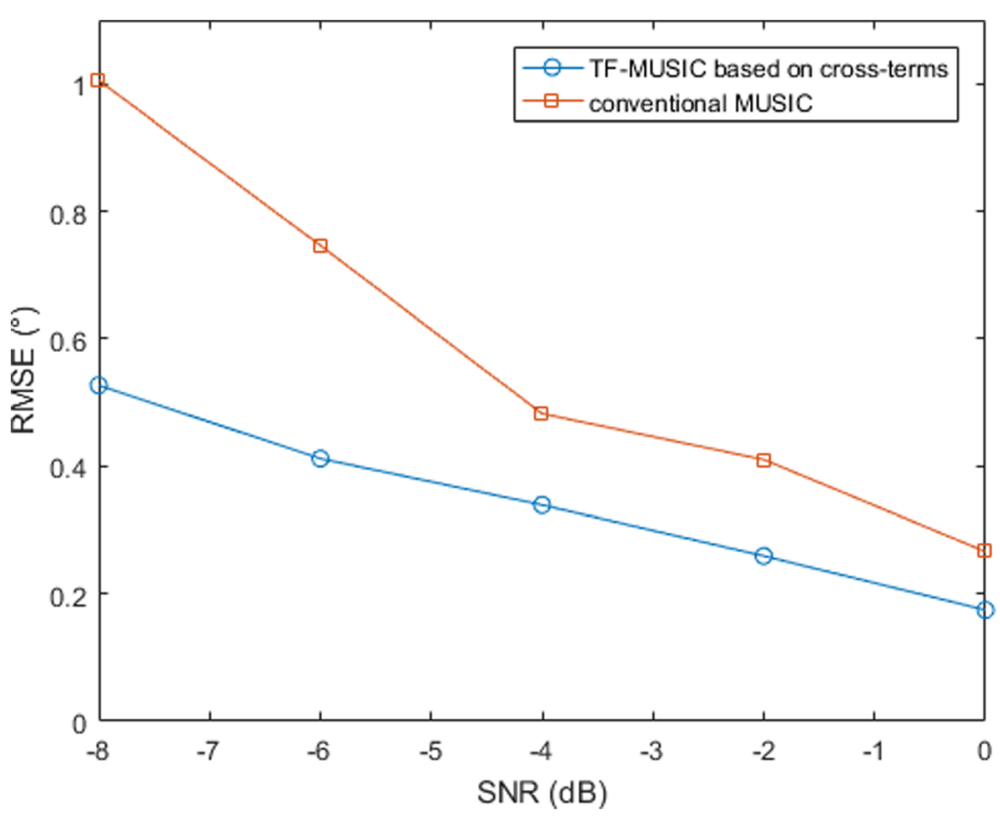

Fig. 7 The RMSE of DOA estimation versus input SNR of weak signal 


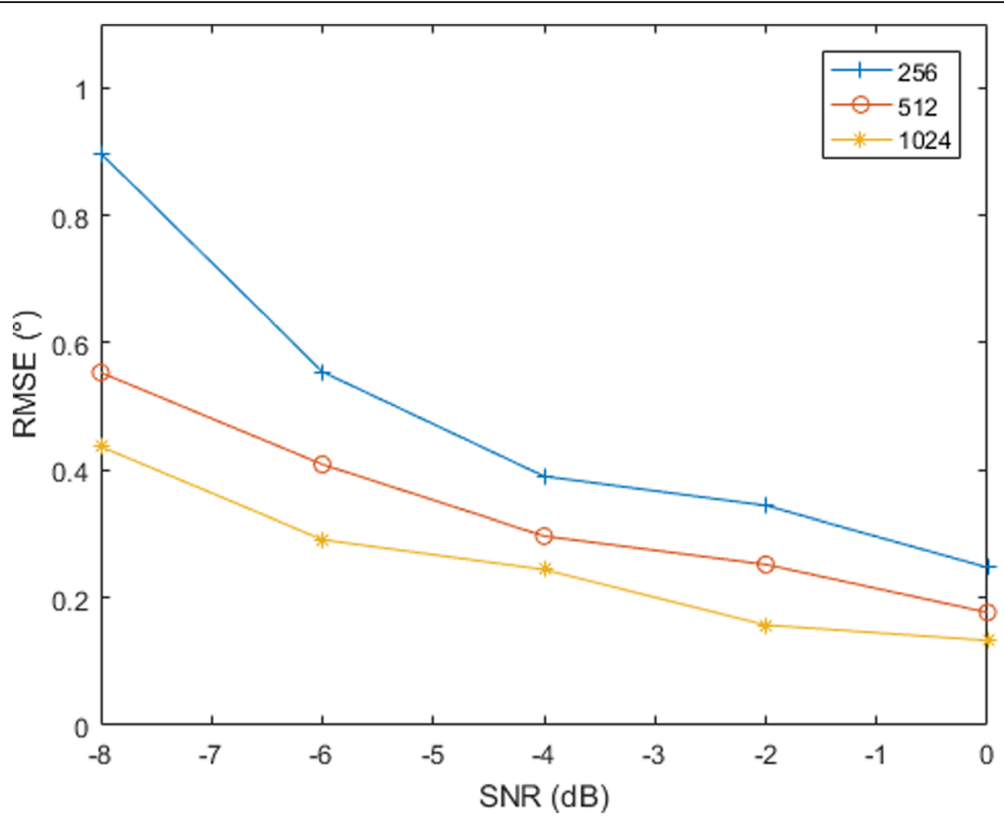

Fig. 8 The RMSE at different snapshots

by the method mentioned above for the DOA estimation. The DOA of the strong and weak signals is simultaneously estimated in Fig. 6, which are approximately $30^{\circ}$ and $40^{\circ}$, respectively. The time-frequency-MUSIC technology is realized respectively for two sets of timefrequency points, each one from one source signal.

When the incoming SNR of $\theta_{1}$ is $5 \mathrm{~dB}$, the input SNR of $\theta_{2}$ is from $-8 \mathrm{~dB}$ to $0 \mathrm{~dB}$. The results were calculated via averaging 100 Monte Carlo runs. Figure 7 shows the DOA estimation root-mean-square error with SNR for traditional MUSIC and time-frequency-MUSIC based on cross-terms. The RMSE of TF-MUSIC based on cross terms is less than that of the conventional MUSIC overall. The advantages of TF-MUSIC based on cross terms in poor SNR conditions become obvious from the figure.

Next, the influence of snapshots on the algorithm is analyzed. With the other simulation conditions mentioned above unchanged, the number of snapshot is 256 ,

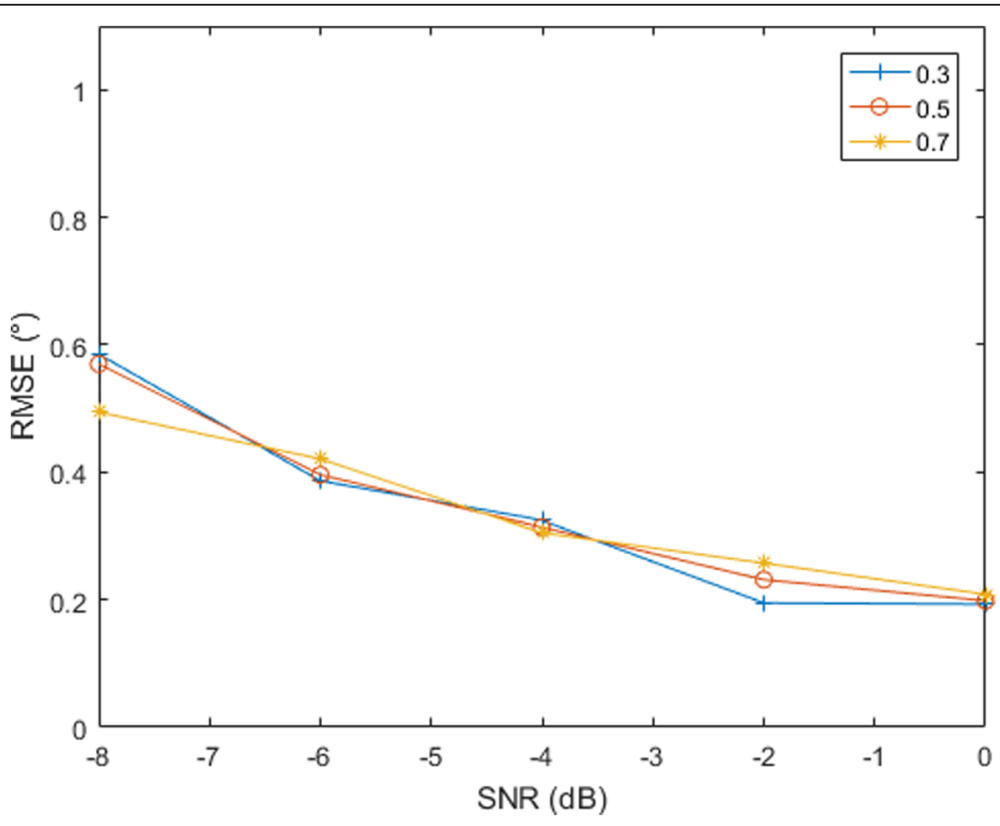

Fig. 9 The RMSE at different $\varepsilon$ 


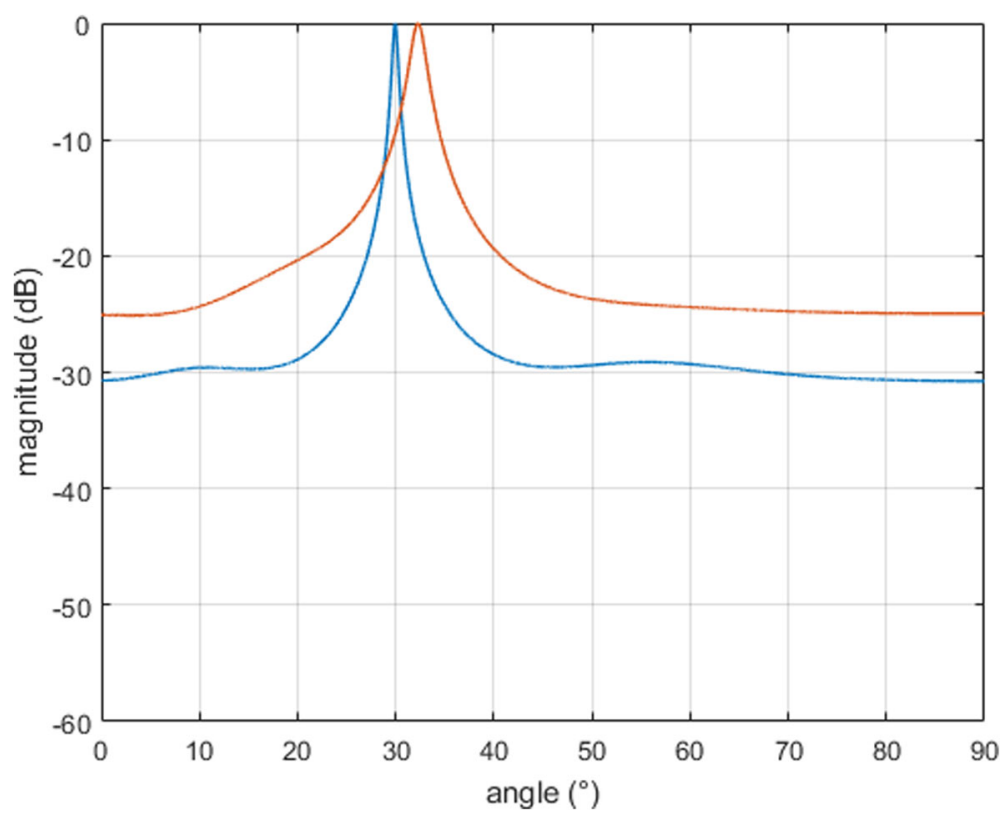

Fig. 10 The spatial spectra of TF-MUSIC

512, and 1024, respectively. From Fig. 8, the increase in the number of snapshots reduces the RMSE of DOA estimation. Then, the increase in that will also increase the calculation time. The impact of a small user-defined positive scalar $\varepsilon$ on RMSE is also simulated. With the 512 snapshots, $\varepsilon$ is $0.3,0.5$, and 0.7 , respectively. From
Fig. 9, different values of the same order have little influence on RMSE.

Figures 10 and 11 show the estimated spatial spectrum of TF-MUSIC based on cross terms and the traditional MUSIC where the direction separation is close $\left(\theta_{1}=30^{\circ}\right.$, $\left.\theta_{2}=33^{\circ}\right)$. It is obvious that the source signals can be

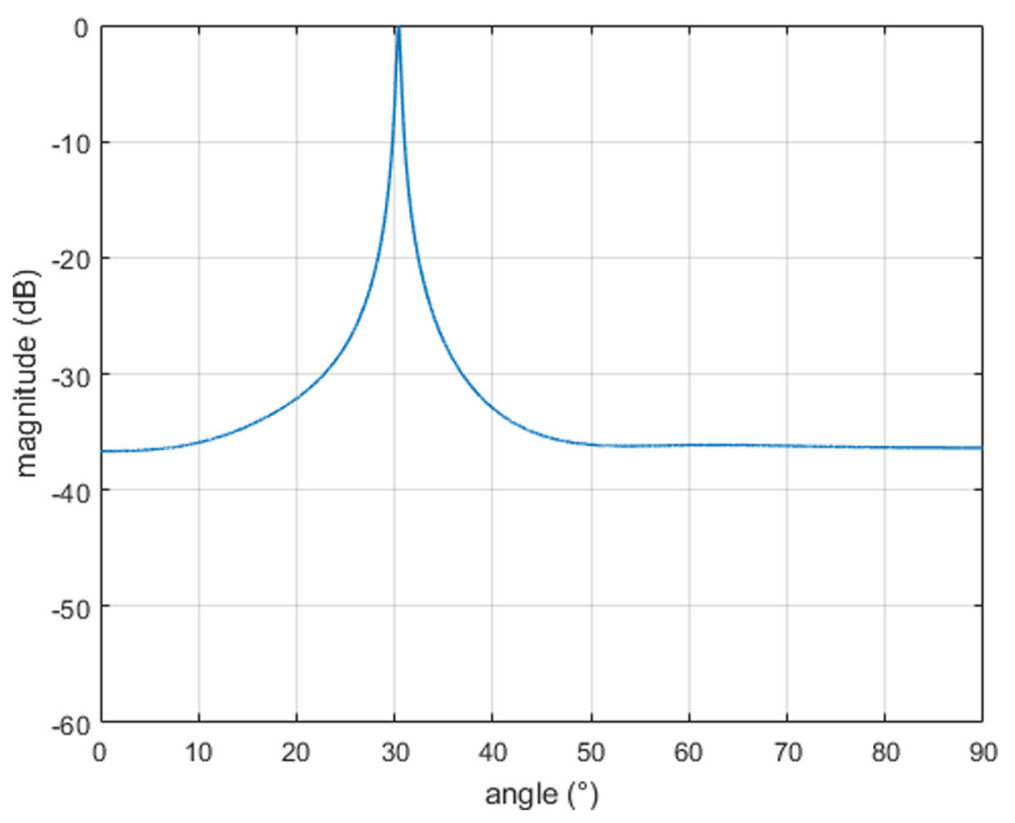

Fig. 11 The spatial spectra of MUSIC 
separated via the time-frequency-MUSIC based on cross terms whereas the conventional MUSIC fails. This is attributed to the combination of timefrequency analysis method and MUSIC algorithm. In the time-frequency MUSIC algorithm, for each signal energy distribution, the MUSIC algorithm is calculated separately, and only a single signal is included in the data covariance matrix. Therefore, two curves are generated and the target with an angle approaching can be distinguished.

\section{Conclusions}

When the desired weak non-stationary signal may be buried in noise, especially in the condition of low signalto-noise ratio, it is difficult or almost impossible to extract the auto-term of the weak non-stationary signal. However, the cross terms of the strong and weak nonstationary signal did not decrease significantly, which contain sufficient information of weak non-stationary signal for its DOA estimation. Therefore, in the case that both strong and weak non-stationary signals exist, meanwhile, the cross terms of STFDs are used to obtain DOA of the desired weak non-stationary signal in this paper. The DOA estimation root-mean-square error of TFMUSIC based on cross terms is less than that of conventional MUSIC. The DOA of two closely spaced signals is resolved by the TF-MUSIC based on cross terms.

\begin{abstract}
Abbreviations
DOA: Direction of arrival; $H T$ : Hough transform; IF: Instantaneous frequency; LFM: Linear frequency modulation; MUSIC: Multiple signal classification; RMSE: Root-mean-square error; SHR-TFRR: Smoothed high-resolution timefrequency rate representation; SNR: Signal-to-noise ratio; STFD: Spatial timefrequency distribution; T-F: Time-frequency; WVD: Wigner-Ville distribution
\end{abstract}

\section{Acknowledgements \\ This work was supported in part by the National Key R\&D Program of China under Grant No. 2017YFC1405202, in part by the National Natural Science Foundation of China under Grant No. 61571157 and Grant No. 61571159, in part by the Public Science and Technology Research Funds Projects of Ocean under Grant No. 201505002, in part by the Natural Science Foundation of Shandong Province under Grant No. ZR2018PF001, and in part by the Foundation of Science and Technology on Communication Networks Key Laboratory.}

\section{Authors' contributions}

SS and AL conceived and designed the experiments; SS and HY performed the experiments; BL contributed simulation tools; and SS and HY wrote the paper. All authors have read and approved the final manuscript.

\section{Funding}

National Key R\&D Program of China under Grant No. 2017YFC1405202 is supporting the data acquisition devices and materials; National Natural Science Foundation of China under Grant No. 61571157 and Grant No. 61571159 are supporting the simulations; the Public Science and Technology Research Funds Projects of Ocean under Grant No. 201505002, the Natural Science Foundation of Shandong Province under Grant No. ZR2018PF001, and the Foundation of Science and Technology on Communication Networks Key Laboratory are supporting the data analyses.

\section{Availability of data and materials}

The datasets used and/or analyzed during the current study are available from the corresponding author on reasonable request.

\section{Competing interests}

The authors declare that they have no competing interests.

\section{Author details}

${ }^{1}$ School of Information Science and Engineering, Harbin Institute of Technology (Weihai), Weihai, China. ${ }^{2}$ Science and Technology on Communication Networks Laboratory, Shijiazhuang, China. ${ }^{3}$ The 54th Research Institute of China Electronics Technology Group Corporation, Shijiazhuang, China.

Received: 10 May 2019 Accepted: 11 September 2019

Published online: 25 October 2019

\section{References}

1. Y. Wei, Z. Zhang, T. Yang, in IET International Conference on Wireless IET. Radar signal processing based on a new cross-term reduced Wigner-Ville distribution (2014)

2. Z. Na, Y. Wang, X. Li, Subcarrier allocation based simultaneous wireless information and power transfer algorithm in $5 \mathrm{G}$ cooperative OFDM communication systems. Phys. Commun. 29, 164-170 (2018)

3. Z. Na, Z. Pan, M. Xiong, et al., Turbo receiver channel estimation for GFDMbased cognitive radio networks. IEEE Access 6, 9926-9935 (2018)

4. G. Gaunaurd, H. Strifors, Signal analysis by means of time-frequency (Wigner-type) distributions-applications to sonar and radar echoes. Proc IEEE 84(9), 1231-1248 (1996)

5. Y. Lai, H. Zhou, Y. Zeng, et al., Quantifying and reducing the DOA estimation error resulting from antenna pattern deviation for direction-finding $\mathrm{HF}$ radar. Remote Sens. 9(12), 1285 (2017)

6. L. Cohen, Time-frequency analysis (Prentice Hall, Englewood Cliffs, 1995)

7. A. Belouchrani, M. Amin, Blind source separation based on time-frequency signal representations. IEEE Trans. Signal Process. 46(11), 2888-2897 (2002)

8. Y. Zhang, W. Ma, M. Amin, Subspace analysis of spatial time-frequency distribution matrices. IEEE Trans. Signal Process. 49(4), 747-759 (2001)

9. W. Mu, M. Amin, Y. Zhang, Bilinear signal synthesis in array processing. IEEE Trans. Signal Process. 51(1), 90-100 (2001)

10. A. Erdogan, T. Gulum, L. Durak-Ata, et al., FMCW signal detection and parameter extraction by cross Wigner-Hough transform. IEEE Trans. Aerosp. Electron. Syst. 53(1), 334-344 (2017)

11. L. Cohen, Time-frequency distributions-a review. Proc. IEEE 77(7), 941-981 (1989)

12. A. Belouchrani, M. Amin, N. Thirion-Moreau, et al., Source separation and localization using time-frequency distributions: an overview. IEEE Signal Process. Mag. 30(6), 97-107 (2013)

13. A. Belouchrani, M. Amin, Time-frequency MUSIC. IEEE Signal Process. Lett. 6(5), 109-110 (2002)

14. S. Barbarossa, A. Zanalda, in IEEE International Conference on Acoustics. A combined Wigner-Ville and Hough transform for cross-terms suppression and optimal detection and parameter estimation (1992)

15. W. Mark, Spectral analysis of the convolution and filtering of non-stationary stochastic processes. J. Sound Vib. 11(1), 19-63 (1970)

16. A. Poyil, S. Meethal, in International Conference on Industrial Control \& Electronics Engineering. Cross-term reduction using wigner hough transform and back estimation (2012)

17. J. Wu, J. Chen, P. Zhong, Time frequency-based blind source separation technique for elimination of cross-terms in Wigner distribution. Electron. Lett. 39(5), 475 (2003)

18. J. Guo, X. Zeng, Z. She, Blind source separation based on high-resolution time-frequency distributions. Comput. Electr. Eng. 38(1), 175-184 (2012)

19. Q. Li, T. Zhou, W. Wang, in IET International Conference on Wireless. New method to eliminate cross-term in wigner distribution (2009)

20. L. Zuo, M. Li, Z. Liu, et al., A high resolution time-frequency rate representation and the cross term suppression. IEEE Trans. Signal Process. 64(10), 2463-2474 (2016)

21. L. Zuo, M. Li, X. Xia, New smoothed time-frequency rate representations for suppressing cross terms. IEEE Trans. Signal Process. 65(3), $733-747$ (2017)

22. D. Aiordachioaie, T. Popescu, in 2017 International Symposium on Signals, Circuits and Systems (ISSCS). A method to detect and filter the cross terms in the Wigner-Ville distribution (2017), pp. 1-4

23. Y. Wu, X. Li, Elimination of cross-terms in the Wigner-Ville distribution of multi-component LFM signals. IET Signal Process. 11(6), 657-662 (2017) 
24. M. Sandsten, J. Brynolfsson, in 2017 25th European Signal Processing Conference (EUSIPCO). Classification of bird song syllables using Wigner-Ville ambiguity function cross-terms (2017), pp. 1739-1743

25. M. Reyna-Carranza, L. Fierro, M. Bravo-Zanoguera, in 2012 Pan American Health Care Exchanges. Wigner distribution's cross terms characterization to detect patterns of ventricular late potentials (2012)

26. J. Jeong, W. Williams, Mechanism of the cross-terms in spectrograms. IEEE Trans. Signal Process. 40(10), 2608-2613 (1992)

27. A. Belouchrani, K. Abed-Meraim, M. Amin, et al., in IEEE International Conference on Acoustics. Joint anti-diagonalization for blind source separation (2001), pp. 2789-2792

28. E. Fadaili, N. Moreau, E. Moreau, Nonorthogonal joint diagonalization/zero diagonalization for source separation based on time-frequency distributions. IEEE Trans. Signal Process. 55(5), 1673-1687 (2007)

29. A. Liu, F. Li, S. Chen, et al., in 2015 Asia-Pacific Microwave Conference (APMC). DOA estimation based on cross terms of spatial time-frequency distribution matrices (2015)

30. R. Schmidt, Multiple emitter location and signal parameters estimation. IEEE Trans. Antennas Propag. 34(3), 276-280 (1986)

31. G. Golub, C. Van Loan, Matrix computations. Math. Gaz. 47(5 Series II), 392$396(1996)$

\section{Publisher's Note}

Springer Nature remains neutral with regard to jurisdictional claims in published maps and institutional affiliations.

\section{Submit your manuscript to a SpringerOpen ${ }^{\circ}$ journal and benefit from:}

- Convenient online submission

- Rigorous peer review

- Open access: articles freely available online

- High visibility within the field

- Retaining the copyright to your article

Submit your next manuscript at $\boldsymbol{\nabla}$ springeropen.com 